\title{
GENERALDIREKTOR DES ÖSTERREICHISCHEN STAATSAR- CHIVS 1994 BIS 2011: LORENZ MIKOLETZKY BEANTWORTET 10 FRAGEN VON BRUNO BAUER
}

Zusammenfassung: Lorenz Mikoletzky beantwortet Fragen über seinen Weg von der Berufsausbildung bis zur Bestellung als Generaldirektor des Österreichischen Staatsarchivs, das er 17 Jahre lang geleitet hat. Thematisiert werden auch die Digitalisierung von Archivgut, das geplante „Haus der Geschichte" sowie Aufgabe und Bedeutung der Historikerkommission, deren stellvertretender Vorsitzender er gewesen ist. Mikoletzky spricht auch sein freundschaftliches Verhältnis zur Vereinigung Österreichischer Bibliothekarinnen und Bibliothekare an sowie sein Bemühen um eine Kooperation von Bibliotheken, Archiven und Museen in der Zusammenarbeitsplattform BAM Austria.

Schlüsselwörter: Österreichisches Staatsarchiv; Haus der Geschichte (Österreich); Digitalisierung; Historikerkommission (1998-2003); Vereinigung Österreichischer Bibliothekarinnen und Bibliothekare; VÖB; BAM Austria; Lorenz Mikoletzky; Generaldirektor 1994-2011; Interview

\section{DIRECTOR-GENERAL OF THE AUSTRIAN STATE ARCHIVES FROM 1994 TO 2011: LORENZ MIKOLETZKY ANSWERING 10 QUESTIONS FROM BRUNO BAUER}

Abstract: Lorenz Mikoletzky answers questions about his professional training and his appointment als Director General of the Austrian State Archives, which he managed for seventeen years. Also discussed are themes like digitalisation of archives, the projected „House of History" and the tasks of dhe Austrian Historical Commission, of which he was Vice-Chairman from 1998 to 2003. Mikoletzky refers also his friendly relations tot he Association of Austrian Librarians and his efforts for a cooperation between libraries, archives und museums in the cooperation plattform BAM Austria.

Keywords: Austrian State Archives; House of History (Austria); digitization; Austrian Historical Commission (1998-2003); Association of Austrian Librarians; BAM Austria; BAM Austria; Lorenz Mikoletzky; Director-General of the Austrian State Archives 1994-2011; interview 


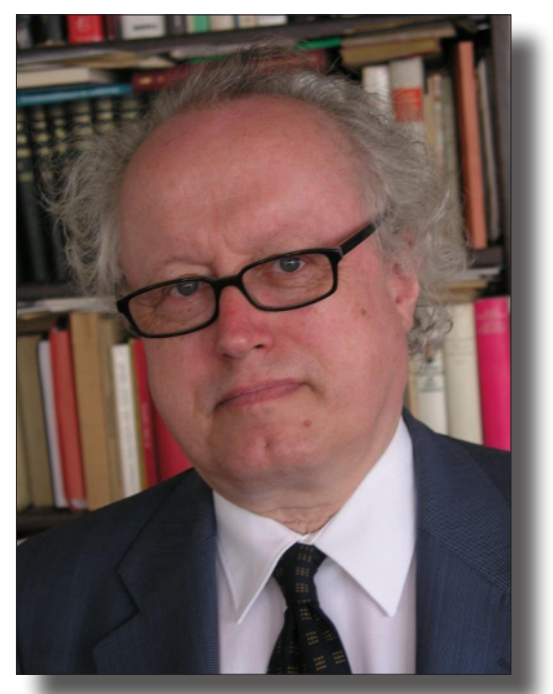

Abb. 1: Dr. Lorenz Mikoletzky, Generaldirektor des Österreichischen Staatsarchivs 1994-2011

\section{1) Archivar}

Bauer: Sie haben 1969 ihre erste Stelle als Archivar am Österreichischen Staatsarchiv angetreten. Welche Motive waren für Sie ausschlaggebend, diese Laufbahn einzuschlagen?

Mikoletzky: Bei der Beantwortung möchte ich in der Familiengeschichte zurückgehen, die auch für weitere Punkte dieses Fragebogens von Relevanz ist. Schlicht und einfach bin ich erblich vorbelastet.

Mein Vater bekleidete, mit Unterbrechung durch den Nationalsozialismus bedingt, die Stelle eines Bibliothekars an der seinerzeitigen Technischen Hochschule und wechselte 1947 in das zwei Jahre zuvor gegründete Österreichische Staatsarchiv. Er war nicht nur Absolvent des Instituts für österreichische Geschichtsforschung, sondern hatte auch die Bibliothekarsprüfung abgelegt. Somit waren diese beiden Bereiche gleichsam von Jugend an meine Begleiter im Alltag.

Nach meinem Studium der Geschichte und der Archäologie sowie ebenso der Ablegung der Staatsprüfung am erwähnten Institut, ergab sich die Möglichkeit in die Archivarslaufbahn einzutreten, was ich sehr gerne tat, zumal mein zweiter Interessensschwerpunkt, die Mittelalterarchäologie, zu dieser Zeit noch in ihren Anfängen lag, was allfällige Berufsaus- 
sichten betraf. Aber auch als Archivar ist man gleichsam ein „Ausgräber“ der früheren Menschheitsgeschichte und kann somit beide Interessensgebiete Archivar/Ausgräber bestens miteinander verbinden, was ich auch 41 Jahre lang versucht habe.

\section{2) Generaldirektor des Staatsarchivs}

Bauer: Nachdem Sie bereits 1991 zum Direktor des Allgemeinen Verwaltungsarchivs ernannt worden waren, erfolgte 1994 Ihre Ernennung zum Generaldirektor des Österreichischen Staatsarchivs. Welche Aufgaben hat man als Generaldirektor des Staatsarchivs?

Mikoletzky: Nachdem ich in der „Wirtschaftsabteilung“ des Österreichischen Staatsarchivs, dem Finanz- und Hofkammerarchiv begonnen hatte, - was mich nebenbei zur näheren Beschäftigung mit dem Dichter Franz Grillparzer als Beamter brachte, war dieser doch 1832-1856 dessen Direktor -, wechselte ich in die innenpolitische Abteilung, das Allgemeine Verwaltungsarchiv, mit dem Zuständigkeitsbereich Kultur, d.h. dem Schwerpunkt Unterrichtsbestand sowie zeitgeschichtlicher Belange. Ungemein reizvolle Bestände waren hier zu betreuen, aus dessen Materialien man für die Forscherinnen und Forscher, aber auch für sich selbst viel lernen kann.

Die Berufung in das Amt des Generaldirektors des Österreichischen Staatsarchivs fasste ich als große Ehre auf, konnte man von dieser Position aus sowohl intern, als auch extern einiges bewegen. In erster Linie lag und liegt die Koordinierung der Arbeit der fünf Archivabteilungen (Haus-, Hofund Staatsarchiv, Allgemeines Verwaltungsarchiv, Finanz- und Hofkammerarchiv, Kriegsarchiv und Archiv der Republik) bei der Generaldirektion. Gegen Ende meiner Tätigkeit erfolgte auf Wunsch des damaligen Bundeskanzlers die Auflösung der Eigenständigkeit des Finanz- und Hofkammerarchivs und dessen Integrierung in das Allgemeine Verwaltungsarchiv. Divergierende Aufgaben waren unter einen Hut zu bringen und das Personal ruhig durch alle Fährnisse, die von außen an das Archiv getragen wurden, zu führen. Ob dies auch in der von mir gedachten Form gelungen ist, müssen andere beurteilen.

\section{3) Highlights}

Bauer: Sie waren 17 Jahre lang Generaldirektor des Österreichischen Staatsarchivs. Was waren für Sie die Highlights in diesen Jahren? 
Mikoletzky: Als die wohl bedeutendsten Highlights meiner Tätigkeit als Generaldirektor möchte ich die 50-Jahrfeier des Österreichischen Staatsarchivs 1995 im Festsaal der Österreichischen Akademie der Wissenschaften mit einer Ansprache des obersten Dienstherren des Archivs, Bundeskanzler Dr. Franz Vranitzky und der Festrede von Univ.-Prof. Dr. Erika Weinzierl, selbst ursprünglich Archivarin am Haus-, Hof- und Staatsarchiv, mit zahlreichen Archivarinnen und Archivaren aus dem In- und Ausland, bezeichnen sowie die Veranstaltung des 15. Internationalen Archivkongresses (2004) in Wien. Hier durften über 2.500 Gäste begrüßt werden, denen ein reiches wissenschaftliches und kulturelles Programm (Ball im Rathaus, von dem noch heute im Ausland erzählt wird) geboten werden konnte. Bei beiden Großereignissen war das gesamte Österreichische Staatsarchiv in Organisation und Durchführung eingebunden, wofür noch heute zu danken ist.

\section{4) Enttäuschungen}

Bauer: Neben vielem Positivem, das Sie als Generaldirektor erleben durften, gab es im Laufe Ihrer langen Karriere wohl auch gelegentlich Enttäuschungen. Wie sehen Sie diese mit einigen Jahren Abstand?

Mikoletzky: Enttäuschungen im wahrsten Sinn des Wortes gab es in den 14 Jahren als Generaldirektor eigentlich keine, zumal ich mich nach dem Spruch richtete, der gerahmt auf meinem Schreibtisch stand und steht: „Nicht ärgern, nur wundern“.

\section{5) Digitalisierung}

Bauer: Digitalisierung ist mittlerweile ein Schlagwort, das bereits viele Bereiche des Alltags erreicht hat. Die Digitalisierung von Beständen wird mittlerweile auch von Archiven - wie auch von Bibliotheken und Museen - in zunehmenden Maße gefordert, wobei einerseits mit der orts- und zeitungebundenen Benutzungsmöglichkeit, andererseits mit der Einsparungsmöglichkeit von Stellfächen für die Aufbewahrung von physischen Objekten argumentiert wird. Wie sollen sich aus Ihrer Sicht Verantwortliche im Bereich der Kulturvermittlung und -bewahrung dieser Herausforderung stellen?

Mikoletzky: Was die Digitalisierung von Archivgut betrifft, wurden diese Überlegungen schon sehr bald an das Österreichische Staatsarchiv herangetragen und fielen von Anfang an auf fruchtbaren Boden, auch wenn gele- 
gentlich Meinungen durch den Raum geisterten, dass nach durchgeführter Arbeit die Originale vernichtet würden.

Natürlich ist die Frage der Erhaltungsdauer der Digitalisate, was auch auf den elektronischen Akt zutrifft, dabei von größter Bedeutung, schreitet hier doch die Entwicklung der Technik rasant voran, der sicherlich im Bereich aller Dokumentationsstellen nicht so einfach nachgekommen werden kann.

\section{6) Haus der Geschichte}

Bauer: Ein großes Thema in Österreich ist derzeit die Planung für ein „Haus der Geschichte" (HdG). Wie beurteilen Sie dieses Projekt, das schon sehr lange diskutiert wird, jetzt aber ein Stadium erreicht hat, in dem nunmehr eine Umsetzung sehr realistisch scheint? Welche Aspekte sollten unbedingt berücksichtigt werden, damit das HdG eine Erfolgs-Geschichte wird.

Mikoletzky: Die Überlegungen, einem „Haus der Geschichte“ auch in unserem Land einen Platz zu verschaffen sind schon relativ lange in der allgemeinen und der politischen Diskussion. Ich erinnere mich an mehrere, schon ins Detail gehende Überlegungen, was etwa einen Bau betraf. Während meiner Aktivzeit nahm ich mit nicht wenigen Vertretern der Universitäten, Archive, Museen und Bibliotheken an Sitzungen zur Einrichtung einer derartigen Institution teil. Alle diese Pläne wurden weitergereicht, kursierten in den verschiedensten Gremien, wurden evaluiert und... verschwanden meist in irgendwelchen ministeriellen (?) Schubladen.

Die Idee als solche ist zu begrüßen, wenn genau feststeht, wohin der Weg führen soll. Das beginnt vor allem mit der Abklärung, was will man mit diesem „Haus". Soll es ein weiteres Museum werden, soll es eine Forschungsstätte für jedermann werden, welche Geschichtsauffassung wird hier nahegebracht und wann beginnt diese österreichische Geschichte. In einem Land, wo der Geschichtsunterricht an den Schulen immer kleiner geschrieben wird und die Studentinnen und Studenten dieses Faches an den Universitäten immer weniger von der Vergangenheit Österreichs wissen, wird der Vermittlung die größte Aufgabe zufallen.

Ein „Haus der Geschichte“ sollte jedenfalls weder einer schon existierenden Organisation untergeordnet werden, - allfälliges Ersparungsdenken greift hier überhaupt nicht -, und dürfte nicht auf (Platz-)Kosten schon existierender Einheiten ins Leben gerufen werden, was in diesem Land bedauerlicherweise gelegentlich vorkommt. 


\section{7) Historikerkommission}

Bauer: Vermögensentzug während der NS-Zeit sowie mögliche Entschädigungsmaßnahmen für Opfer dieses Unrechts waren in Österreich sehr lange zu wenig beachtete Themen. Dieses Versäumnis wurde erst durch die zwischen 1998 und 2003 tätige Historikerkommission der Republik Österreich, deren stellvertretender Vorsitzender Sie waren, behoben. Was war die Aufgabe dieser Kommission, und wie schätzen Sie zwölfJahre nach Übergabe des Abschlussberichts - deren Erfolg heute ein?

Mikoletzky: Wie allgemein bekannt, ist der Umgang Österreichs mit seiner Geschichte der Ersten Republik und der NS-Zeit immer wieder ein schwieriger. Die Historikerinnen und Historiker, die diese Zeiträume aufarbeiteten, waren sogar gelegentlich von der eigenen Zunft nicht gerne gesehen. Als 1998 die „Historikerkommission“ von der Politik ins Leben gerufen wurde, war dies ein sehr bedeutender Moment. Ich sah meine Aufgabe als stellvertretender Vorsitzender und Vertreter des Archivwesens in diesem Gremium besonders als Hilfestellung seitens der Institutionen, ohne die Aufarbeitung von Geschichte unmöglich ist. Die Behandlung der Thematik des Vermögensentzuges sowie allfälliger Rückstellungen und Entschädigungen, die in insgesamt 54 Berichten ihren Niederschlag fanden, war für meine Person gelegentlich sehr emotionsreich. Es sollte jedoch mit dieser Kommission kein Schlussstrich unter die Aufarbeitungen all dieser Probleme gezogen, sondern der zukünftigen Forschung noch viel Platz für weitere Recherchen gelassen werden.

Ein besonderer Aspekt für die Archivforschung ergab sich im Laufe der Untersuchungen: 2000 trat erstmals ein staatliches Archivgesetz in Kraft, nachdem die Jahre seit Gründung des Österreichischen Staatsarchivs 1945 immer nur mit Einzelerlässen des Bundeskanzleramtes gearbeitet worden war. Dieses Archivgesetz gab in der Folge auch den Bundesländern Anlass eigene Landesarchivgesetze auszuarbeiten, die sich in vielen Dingen am Bundesgesetz orientieren. Rückblickend würde ich sagen, dass die Kommission sehr gute, wichtige Arbeit geleistet und für die zukünftigen Forschungen zur NS-Zeit und ihren Folgen in Österreich wesentlichste Weichen gestellt hat.

\section{8) VÖB}

Bauer: Sie haben sich während Ihrer gesamten beruflichen Laufbahn auch immer darum bemüht, dass Archivarinnen und Archivare sowie Bibliothekarinnen und Bibliothekare ein gegenseitiges Verständnis entwickeln. In diesem Sinn haben Sie sich über 
Jahre als führender Archivar in Österreich auch in der Vereinigung Österreichischer Bibliothekarinnen und Bibliothekare engagiert, wo Sie auch derzeit noch im Vorstand mitarbeiten. Auch in der Bibliothekarsausbildung haben Sie als Vortragender Generationen von Bibliothekarinnen und Bibliothekare in die Grundzüge des Archivwesens eingeführt. Was waren und sind Ihre Beweggründe für die Übernahme dieser „archivfremden" Aufgaben?

Mikoletzky: Die beiden Berufsgruppen Archivare/Bibliothekare sind einerseits enger verbunden, als gelegentlich angenommen wird, andererseits doch sehr verschieden. Einerseits wird ungedrucktes Schriftgut als Unikat verwaltet und dies oftmals bis in früheste Tage der Schriftlichkeit, andererseits das Gedruckte, das auch anderen Inventarisierungsvorgaben unterliegt, als Archivalien. Und doch war viele Jahre vor dem Zweiten Weltkrieg die Ausbildung beider Berufsgruppen gemeinsam, sodass man voneinander mehr wusste, als dies vielleicht in breitesten Kreisen bekannt war. Das Institut für österreichische Geschichtsforschung behielt das Fach Bibliothekskunde noch bis gegen Ende des 20. Jahrhunderts in seinem Lehrplan, die Bibliothekare führten die Information zur Archivistik ein. Und bei diesen Reformüberlegungen habe ich mich von Anfang an eingebracht, um die Unterschiede zwar nicht einfach zu negieren, aber doch mit Hilfe der Gemeinsamkeiten der Wissenschaft dienlich sein zu können.

Dass ich im VÖB-Vorstand als (einziger) Archivar vertreten bin, ruft kein Minderwertigkeitsgefühl hervor. Ich fühlte mich immer als Gleicher unter Gleichen und dass mir die Ehrenmitgliedschaft für die Verbindung von der VÖB mit dem Archivwesen und gelegentliche Hilfe bei historischen Klärungen verliehen wurde, war für mich ein ganz besonderer Aspekt in meinem Leben, für den ich sehr dankbar und auf den ich sehr stolz bin.

\section{9) BAM}

Bauer: Auch BAM Austria, die informelle Kooperationsplattform von Bibliotheken, Archiven und Museen in Österreich, ist Ihnen ein großes Anliegen. So fanden etwa die regelmäßigen Sitzungen von BAM Austria über Jahre in dem von Ihnen als Generaldirektor geleiteten Staatsarchiv in Wien-Erdberg statt. Warum gibt es BAM Austria? Wo sehen Sie Bereiche, in denen Bibliotheken, Archive und Museen (stärker) zusammenarbeiten sollten?

Mikoletzky: Im Blick auf das vorher Gesagte erscheint es eigentlich klar, dass die an mich herangetragene Idee der Beteiligung an der Schaffung von 
BAM auf fruchtbaren Boden fiel und ich seither, auch im „Unruhestand“ seit 1. Jänner 2012, bemüht bin, dieser Zusammenarbeitsplattform der drei Bereiche Bibliotheken, Archive und Museen Leben zu geben. Es ist dies nicht immer sehr einfach. Nicht dass die Aufgaben der Drei zu divergent wären, nein, bedauerlicherweise ist das Interesse an der Mitwirkung wie man so sagt nicht gerade „abendfüllend“. Dabei gibt es etwa in Deutschland ein spiegelgleiches Gremium, das recht aktiv ist.

Die Aufgabenvielfalt, die schon besprochen wurde oder in der Zukunft auf dem Programm stehen sollte, betrifft alle drei „Institutionen“, wenn etwa über das Urheberrecht gesprochen wurde oder die schon angesprochene Digitalisierung als Problematik zu betrachten wäre.

Der diesjährige Bibliothekarstag zur Thematik „offen(siv)e Bibliotheken" wird erstmals Gelegenheit bieten, einem breiten Publikumskreis BAM näher zu bringen, und ich erwarte dabei interessierte Reaktionen, die die Arbeit des Gremiums für die weitere Zukunft beflügeln werden.

\section{0) Zukunft der Gedächtnisinstitutionen}

Bauer: Sie haben jahrelang das größte Archiv des Landes geleitet und dabei auch die Entwicklung an wissenschaftlichen Bibliotheken mitverfolgt. Würden Sie aufgrund Ihrer Erfahrungen jungen Menschen heute empfehlen, einen Arbeitsplatz in einem Archiv oder einer Bibliothek anzustreben? Und wenn Sie diese Frage - wie ich mir erhoffe bzw. wohl erwarten darf - mit Ja beantworten, welche Voraussetzungen sollten Sie mitbringen?

Mikoletzky: Diese Frage kann ich wirklich guten Gewissens mit „Ja“ beantworten. Es wartet in jedem angesprochenen Bereich sehr interessante Arbeit.

Man muss aber Interesse und gewisse Kenntnisse des Berufes mitbringen. Praxis bekommt man im Alltag. Dabei ist im Archivbereich natürlich an erste Stelle das historische Wissen im Allgemeinen und überhaupt im österreichischen Bereich unbedingte Voraussetzung. Eine weit verbreitete Vorstellung sollten alle an Archiv- oder Bibliotheksarbeit Interessierten hintanstellen: Man sitzt doch an den Quellen und kann daher andauernd wissenschaftlich arbeiten. Dies ist ein Irrtum - in erster Linie sind Archive und Bibliotheken Dienstleistungsbetriebe für Benutzer und Benutzerinnen, Amtsstellen etc. Da kommt man bei gewissenhafter Erledigung dieses Aufgabenbereiches sowie der andauernd anfallenden internen Ordnungsarbeiten gar nicht dazu, eigene Arbeiten zu produzieren. Dazu ist die Zeit nach Büroschluss oder an den Wochenenden da. 
Kein Geringerer, als der angesprochene Grillparzer stellte gelegentlich fest, dass das Amt ihm die notwendige Zeit zum Arbeiten stehle. Auch wenn immer wieder der Ruf nach Einsparungen erklingt und gelegentlich die Bedeutung der „Gedächtnisinstitutionen“ eines Landes in Frage gestellt wird, so sollte bei jeder Gelegenheit betont werden, dass ohne die drei Bereiche Archive, Bibliotheken und Museen eine geschichtslose Menschheit existieren würde. Wollen wir das wirklich?

Hon.-Prof. Dr. Lorenz Mikoletzky

Österreichisches Staatsarchiv

Generaldirektor i. R.

E-Mail: lorenz.mikoletzky@oesta.gv.at

Mag. Bruno Bauer Universitätsbibliothek der Medizinischen Universität Wien

Währinger Gürtel 18-20, 1097 Wien E-Mail: bruno.bauer@meduniwien.ac.at

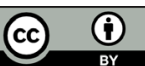

Dieses Werk ist lizenziert unter einer Creative-Commons-Lizenz Namensnennung 4.0 International 